\title{
Die TARMED-Notmassnahmen im Rahmen der Radiologie
}

\author{
F. Bossard, Ressort Tarife SGR-SSR
}

Wie bekannt wurden im Bereiche UV/MV/IV einerseits und im Bereich KVG andererseits für die freipraktizierenden Radiologen Notmassnahmen diskutiert, ausgehandelt und beschlossen sowie eingeführt. Dies bedarf einer Erläuterung.

Korrespondenz:

Dr. med. François Bossard Scherzligweg 16 CH-3600 Thun
Die Fachgesellschaft der Radiologen hat bei der Entwicklung der damaligen GRAT schon sehr früh, seit 1998, und nach Ergänzung zu TARMED erst recht einen sehr rauen Wind verspürt.

Im Rahmen des Reengineerings (RE) wurden die Modellgrundlagen und deren Eckwerte vor allem im INFRA-Modellteil durchleuchtet, und dabei wurde festgestellt, dass in der Tat verschiedene Faktoren zu einem unwirtschaftlichen Tarif geführt haben, der die freipraktizierenden Radiologen innert kürzester Zeit in den Ruin treiben würde. Diese Tatsache haben alle Vertragspartner anerkannt. Deshalb haben wegen der Einführung am 1. Mai 2003 zuerst die FMH mit der MTK und später G7 mit santésuisse die Sache an die Hand genommen, Verträge ausgehandelt und eingeführt.

Da die Neutralitätsvorgaben zwischen UV/ MV/IV mit der Fallkostenstabilisierung anders verlaufen wie die Kostenneutralität im KVG, wurden unterschiedliche Lösungen angestrebt.

Im Fall der Fallkostenstabilität ist der SUVATarif Referenzgrösse, während im KVG-Bereich die Berechnungen und Eckwerte des Reengineerings angenommen wurden, dies obwohl im RE verschiedene Modellkonzepte und Korrekturen noch nicht endgültig berücksichtigt sind und zu vervollständigen wären.

Die bisher von den Notmassnahmen ausgeschlossenen Spitäler haben initial einen anderen Lösungsweg versucht: höherer Taxpunktwert als
Fr. 1.-; weil dies aber von santésuisse abgelehnt wurde, werden nun Lösungen analog der freipraktizierenden Radiologen angestrebt.

Die Einsicht der verhandelnden Radiologen in das Modell TARMED hat gezeigt, dass nicht nur im Bereich der Radiologie verschiedene Modellansätze unrealistisch sind, sondern auch verschiedene Eckwerte:

Kritisch sind die Sach- und Umlagekosten, die Materialkosten und die Auslastungen im Bereiche der TL, ferner die Löhne und Indizes, dann die Dignitäten und Produktivitäten im Bereiche der AL, welche zum Teil nicht einmal auf die Auslastungen der Geräte abgestimmt sind. In diese Problematik kommt die Anwendung der bildgebenden Systeme bei Radiologen und Nichtradiologen. Die Minutagen bedürfen ebenfalls einer Überarbeitung; vor allem in einzelnen Leistungspositionen sind provokativ-störende Einzelwerte eingefügt; dabei ist eine Bewertung im Kontext der Untersuchung und Behandlung vorzunehmen, welche in den Kritiken der Ärzte vielfach nicht berücksichtigt sind. Dies führt unter anderem zu den entsprechenden Verzerrungen und Unwuchten in den Auswertungen der FKS und $\mathrm{KN}$ in laufenden Überwachungen der Assessmentkommission und des KN-Büros. Aus den Erkenntnissen aus den Mengengerüsten und Leistungsprofilen der Ärzte im Abrechnungsverhalten können korrekte Korrekturmassnahmen gewonnen werden.

Es ist zu wünschen, dass sich die Vertragsparteien bald über eine gründliche Überarbeitung von TARMED einigen, damit die sich abzeichnenden Unwuchten auch in anderen Fachbereichen korrigiert werden können. 